\title{
Spatial modelling of brief and long interactions between $T$ cells and dendritic cells
}

\author{
Joost B Beltman, Athanasius FM Marée and Rob J de Boer
}

In the early phases of an immune response, T cells of appropriate antigen specificity become activated by antigen-presenting cells in secondary lymphoid organs. Two-photon microscopy imaging experiments have shown that this stimulation occurs in distinct stages during which T cells exhibit different motilities and interactions with dendritic cells (DCs). In this paper, we utilize the Cellular Potts Model, a model formalism that takes cell shapes and cellular interactions explicitly into account, to simulate the dynamics of, and interactions between, T cells and DCs in the lymph node paracortex. Our three-dimensional simulations suggest that the initial decrease in T-cell motility after antigen appearance is due to 'stop signals' transmitted by activated DCs to T cells. The long-lived interactions that occur at a later stage can only be explained by the presence of both stop signals and a high adhesion between specific T cells and antigen-bearing DCs. Furthermore, our results indicate that longlasting contacts with T cells are promoted when DCs retract dendrites that detect a specific contact at lower velocities than other dendrites. Finally, by performing long simulations (after prior fitting to short time scale data) we are able to provide an estimate of the average contact duration between T cells and DCs.

Immunology and Cell Biology advance online publication, 10 April 2007; doi:10.1038/sj.icb.7100054

Keywords: Cellular Potts Model; DC T-cell interactions; immunological synapse; T-cell motility; two-photon microscopy

The development of adaptive immune responses requires that $\mathrm{T}$ cells of relevant antigen specificity become activated, obtain effector function, and undergo proliferation. This typically occurs in secondary lymphoid organs such as lymph nodes (LN) and spleen, where T cells continuously search for dendritic cells (DCs) that present foreign peptides. Thanks to the application of two-photon microscopy (2PM) to intact, living lymphoid tissues, ${ }^{1-13}$ the behaviour of immune cells and their interactions with other cells can now be visualized directly.

From such imaging experiments, it was discovered that $\mathrm{T}$ cells, in the absence of cognate antigen, crawl around at remarkably high mean velocities of $9-12 \mu \mathrm{m} \mathrm{min}^{-1}$. 1,3,5 They achieve peak velocities of more than $25 \mu \mathrm{m} \mathrm{min}^{-1}$, but they also experience phases of brief 'pausing. Although they walk in a more or less consistent direction over periods of several minutes, they perform a random walk in the longer run. Both $\mathrm{CD}^{+}$and $\mathrm{CD}^{+} \mathrm{T}$ cells exhibit this behaviour, although the trajectories may be biased. Recently, it was shown that the presence of cognate $\mathrm{CD}^{+}{ }^{+} \mathrm{T}$-cell-DC interaction sites assists in guiding $\mathrm{CD}^{+} \mathrm{T}$ cells to such sites by means of chemokines. ${ }^{13}$ Another strategy to increase the probability of T-cell-DC encounters is the dynamic behaviour of DCs: they vigorously extend and retract long dendritic processes, thus greatly increasing the LN volume that DCs are able to scan. ${ }^{4}$ When a T-cell encounters a DC, the ensuing (non-cognate) interaction takes on average approximately $3 \mathrm{~min} .{ }^{1,3,5}$ During such an interaction the Tcell is still able to crawl along the surface of the DC, ${ }^{14}$ presumably scanning it for relevant peptide-major histocompatibility complexes.
The behaviour of $\mathrm{T}$ cells changes dramatically when DCs are presenting cognate antigen, which occurs in several distinct phases. ${ }^{5,14}$ When $\mathrm{T}$ cells first start to detect the presence of DCs presenting antigen of appropriate specificity, their motility decreases ('phase 1') due to a lower mean velocity ${ }^{14}$ and a larger mean turning angle $e^{5,14}$ (turning angle data from Mempel et al. ${ }^{5}$ are replotted in Figure $2 \mathrm{~b}$ and c). The average duration of interactions with DCs increases slightly, but is generally still referred to as brief contacts. Several hours after T cells are first exposed to their cognate antigen, a much more striking shift in their dynamic behaviour takes place ('phase 2'; note that the exact timing depends on the particular experimental circumstances, compare differences in $\left.{ }^{5-7,14}\right)$. Most $\mathrm{T}$ cells are now organized in clusters around DCs and no longer exhibit active movement. Indeed, the duration of interactions increases such that most $\mathrm{T}$ cells (around $80 \%$ ) remain associated with single DCs for longer than the entire visualization period of $30-60$ min..$^{5,14}$ It is likely that immunological synapses (IS) ${ }^{15,16}$ are formed during this phase of long-lasting contacts. Although IS formation has not yet been clearly demonstrated in vivo, the finding that CD43 is excluded from the CD4 ${ }^{+}$T-cell-DC interface ${ }^{17}$ points in the direction of IS structures. Approximately 1 day after T-cell transfer, T cells terminate their long interactions with DCs, resume their rapid movement in the $\mathrm{LN}$, and start proliferating ('phase 3 '). Miller et al. ${ }^{14}$ additionally report an intermediate stage where enlarged T-cell blasts are 'swarming' around DCs, that is, move slowly in looping patterns within a local area. 
The novel in vivo data on the dynamic behaviour of various cell types allows for the construction of detailed models of the processes taking place in LNs. Such modelling should lead to new predictions that can be tested experimentally, and this in turn should result in refinement of the models, that is, a 'marriage' between models and experiments. ${ }^{18,19}$ We constructed previously a spatial model of the LN $\mathrm{T}$-cell area to investigate the default dynamics of naïve $\mathrm{T}$ cells in the absence of cognate antigen. ${ }^{20}$ For this purpose we used a threedimensional (3D) Cellular Potts Model (CPM), ${ }^{21,22}$ in which cells occupy several sites on a 3D lattice, and shape changes that cells undergo while migrating and interacting with other cells are taken into account. The model formalism generally allows for very realistic simulations of cellular dynamics. The CPM is very well suited for making predictions that are testable by new 2PM imaging experiments. This is because the same type of measurements can be performed on simulated cells as on real cells, which allows for a direct comparison, on a matching level of detail, between model and experimental outcome. A good example of the predictive power of the CPM is given by our observation of small, dynamic T-cell streams in our previous simulations. ${ }^{20}$ We confirmed the existence of such T-cell streams in real LNs by performing experiments in which a (relatively) large number of $\mathrm{T}$ cells was visualized. ${ }^{20}$ Finally, an important additional advantage of the CPM is that it can be utilized to extrapolate results to larger scales (e.g., large population of cells or long time period) than accessible with the current experimental techniques.

In this paper, we extend our previous results on T-cell behaviour in the absence of cognate antigen ${ }^{20}$ (summarized below) by investigating which circumstances are required to obtain long-lived interactions between T cells and DCs. We focus on how the migration behaviour of $\mathrm{T}$ cells is affected by changes in their adhesion properties and 'stop signals' transmitted by DCs, as well as by the dynamic behaviour of DC dendrites.

\section{RESULTS}

\section{Modelling interactions between $\mathrm{T}$ cells and DCs}

As in our previous simulation work on the migration behaviour of $\mathrm{T}$ cells in the LN paracortex, we consider a limited, wrapped space filled with static rods, representing the reticular network (RN) created and ensheathed by fibroblastic reticular cells (FRCs), ${ }^{23}$ and extracellular matrix (ECM). Our in silico $\mathrm{T}$ cells and DCs are continuously maneuvering through this space according to well-defined migration rules (described briefly below and in detail in Methods). A small fraction of the DCs bear antigen that is specifically recognized by a small subset of the T cells. The other DCs do not present 'relevant' antigen, and the other, non-specific T cells do not respond to any of the antigens presented by DCs. The characteristics that distinguish these different in silico cell populations include a high adhesion between specific $\mathrm{T}$ cells and antigen-bearing DCs, and 'stop signals' transmitted by antigen-bearing DCs towards specific T cells (hereafter referred to as specific stop signals, as opposed to non-specific signals that would be directed to all $\mathrm{T}$ cells).

Each in silico T-cell is endowed with a polarity that determines its preferred direction of movement. This polarity changes over a period of seconds according to the recent displacement of the cell, which results in a realistic, self-adjusting type of motility (as also shown $\mathrm{in}^{20}$ ). It was recently discovered that lymphocytes have a clear tendency to walk along the RN. ${ }^{12}$ Therefore, simulated $\mathrm{T}$ cells are considered to have a strong preference to adhere to the $\mathrm{RN}$.

We previously constructed in silico DCs by giving them a large surface area to volume ratio. ${ }^{20}$ Although this resulted in small, dynamic tentacles, it was not possible to create long dendrites with this method because a too large surface area to volume ratio leads to frequent breaking off of DC parts. Although undesirable, this was acceptable for the questions addressed in that paper because it involved only brief interactions between $\mathrm{T}$ cells and DCs. However, this approach is problematic when one investigates long-lived interactions because T cells that strongly adhere to DCs start tearing off DC fragments and drag them along on their journey through the simulated LN. Here, we devised another way to create in silico DCs in which dendrite dynamics are explicitly defined (Figure 1a and Supplementary Video S1). We describe bundles of actin that are constantly pushing against the cell membrane in a certain direction (randomly chosen for each new bundle), giving rise to the outgrowth of multiple dendrites. Once a dendrite has experienced a period of extension, it pulls back, and subsequently a new (random) dendrite starts growing. This method solves the problem of DC fragmentation, because the underlying actin bundles cannot break. Our DCs do not exhibit any directional preference. DCs are considered to maintain themselves in a more or less fixed mean position among a network of other DCs and the RN. ${ }^{24}$

\section{Motility of $\mathrm{T}$ cells in the absence of antigen}

When the characteristics of specific and non-specific T cells, as well as those of antigen-bearing DCs and those bearing no relevant antigen, are considered to be the same, we are in fact modelling the situation in the absence of antigen. As we showed previously, ${ }^{20}$ the T-cell dynamic behaviour in this scenario matches experimental imaging results very well (Figure $1 \mathrm{~b}$ and Supplementary Video S2). The in silico $\mathrm{T}$ cells perform a random walk, although their motion is more or less linear on time scales of several minutes. This can be seen from an overlay of their normalized tracks (Figure 1c), and from the relation between their mean displacement and square root of time (Figure 1d). During their journey through the simulated LN, our T cells exhibit realistically large velocity fluctuations (Figure 1e) and they self-organize in dynamic micro-streams (not shown). Further, their brief contacts with DCs last up to maximally $10 \mathrm{~min}$, though most are of much shorter duration (Figure 1f).

As a consequence of the polarity of simulated $\mathrm{T}$ cells, they have a strong preference to continue in an approximately similar direction (i.e., they have a persistence). This can be seen from their turning behaviour (Figure 2a). The distribution of the turning angles is skewed towards smaller angles in comparison with what is to be expected when $\mathrm{T}$ cells would be choosing random turning angles. The dashed line shows the expected distribution. ${ }^{25}$ The shape of the expected distribution for random turning angles stems from the fact that different angles occur with unequal probabilities in three dimensions. For instance, it can be easily seen that there are many ways to make large turns of approximately $90^{\circ}$, whereas there are much less ways to make small turns in 3D. This also explains why the distribution of lymphocyte turning angles exhibits a minimum at $0^{\circ}$. Preston et al. ${ }^{25}$ quantified the T-cell persistence in data by Mempel et al. ${ }^{5}$ (these data are replotted in Figure $2 \mathrm{~b}$ and $\mathrm{c}$ ) by calculating the mean of the cosine of the turning angle $\phi$. In this measure, $\cos \phi=0$ means random turning, whereas $\cos \phi=1$ is equivalent to perfectly persistent behaviour. In the absence of antigenbearing DCs they found $\cos \phi=0.47$, that is, a relatively strong tendency to walk in a consistent direction. In our model, we find that the mean of cosine $\phi=0.82$, which means that the T-cell persistence in our simulations is stronger than that measured experimentally (compare Figure 2a and b; see Discussion for a possible explanation). 


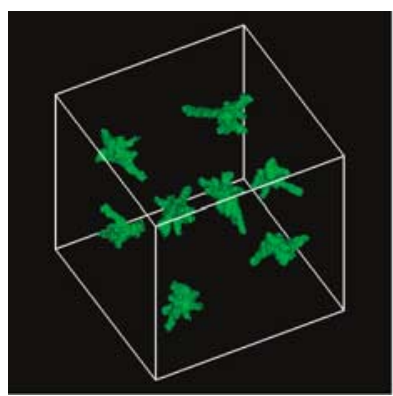

b

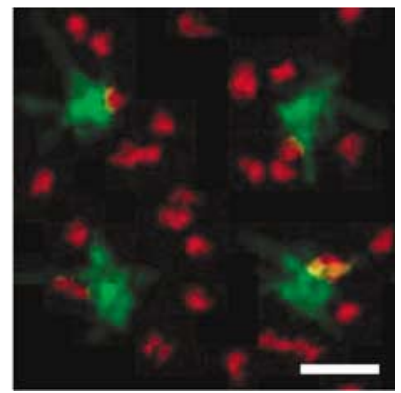

C

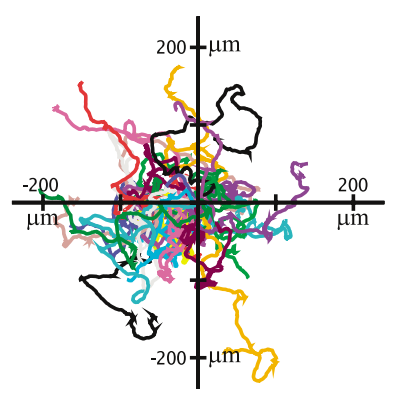

d

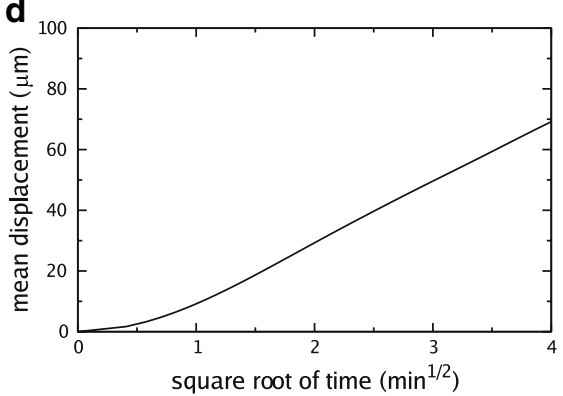

e

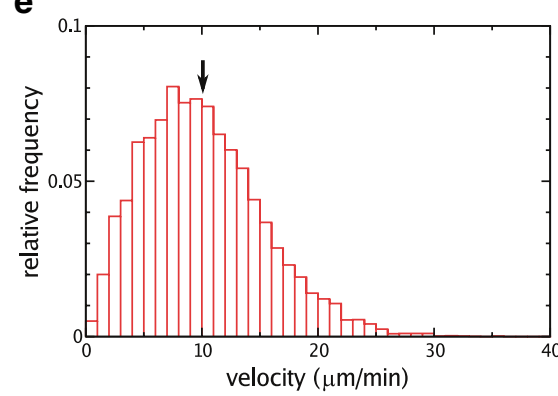

f

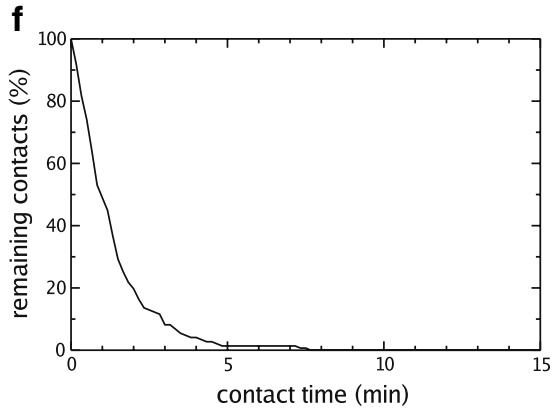

Figure 1 Dynamic behaviour of simulated T cells in the absence of antigen. (a) 3D Snapshot at time 23:10 showing antigen-bearing DCs. (b) Snapshot with compression along the $z$-direction (top-view) at time 00:00 showing specific T cells and antigen-bearing DCs. Scale bar: $20 \mu$ m. (c) Overlay of individual T-cell tracks from a 60-min period in $x y$ coordinates after aligning their starting positions. (d) Mean displacement plot. (e) Distribution of T-cell velocities. Arrow denotes mean velocity $\left(10.1 \mu \mathrm{m} \mathrm{min}^{-1}\right)$. (f) Percentage of contacts between $\mathrm{T}$ cells and DCs that remains in contact after the given duration.

\section{The impact of antigen on T-cell motility}

Next, we investigate how the behaviour of in silico T cells is affected by increasing the preference of specific $\mathrm{T}$ cells to adhere to antigenbearing DCs, as well as by 'stop signals' that are transmitted by antigen-bearing DCs towards specific $\mathrm{T}$ cells (the two effects are investigated both separately and combined). Such specific stop signals are only given when the two cell types are in physical contact, and as a result the particular T cell will lose its persistent movement. Once this contact is broken, the T-cell resumes its high motility.

Assuming that specific $\mathrm{T}$ cells adhere strongly to antigen-bearing DCs (but that these DCs do not transmit stop signals) has only a limited effect on the dynamic behaviour of the T cells (Figure 3 and Supplementary Video S3). It neither affects the motility coefficient (Figure 3a) nor the distributions of velocities (Figure 3c) and turning angles (Figure 3a, c, and d; compare black and red lines). However, the contact duration shows a moderate increase (Figure $3 \mathrm{~b}$ ). Hence, strong adhesion alone can explain slightly longer contacts due to the preference of specific $\mathrm{T}$ cells to remain in contact with antigen-bearing DCs during their voyage. However, because $\mathrm{T}$ cells retain their directional propensity, they do not slow down, or make larger turns, and thus achieve similar motility coefficients as in the low-adhesion case.

When antigen-bearing DCs transmit stop signals to specific $\mathrm{T}$ cells (but there is no strong adhesion between these cell types), this has a clear effect on the T-cell behaviour (Figure 3 green lines, Supplementary Video S4). It results in a decreased motility coefficient (Figure 3a) and mean velocity (Figure 3c), an increased contact duration (Figure $3 \mathrm{~b}$ ), and larger turning angles (Figure $3 \mathrm{~d}$ ) than in a scenario without stop signals. This is because the direction in which a T-cell resumes its directed movement after a stop is environmentally determined and therefore unrelated to its previous direction. Hence, stop signals transmitted by DCs have a more pronounced effect on Tcell motility than a high adhesion between antigen-bearing DCs and specific T cells.
Still, specific stop signals alone are not sufficient to explain longlasting contacts, which is due to the competition by the large number of fast-moving, non-specific T cells. Our simulation outcomes demonstrate that interactions of several hours between T cells and DCs can only be obtained when both specific stop signals are present and the adhesion between specific T cells and antigen-bearing DCs is high (Figure 3 blue lines, Supplementary Video S5). This indeed leads to a very low motility coefficient (Figure 3a) and mean velocity (Figure 3c), and turning angles that nearly follow the distribution that is expected when turning is random (Figure 3d). Furthermore, most contacts stay intact for longer than the time of measurement $(1 \mathrm{~h}$, which is also the typical experimental observation time; Figure $3 \mathrm{~b}$ ).

\section{The dynamic behaviour of dendrites}

2PM imaging experiments have demonstrated that DCs extend and retract their long dendritic processes at high velocities. ${ }^{4}$ Presumably, this strategy increases the rate at which they are able to scan the LN paracortex for specific $\mathrm{T}$ cells. The explicit definition of simulated dendritic processes results in realistic dynamic behaviour of DCs (Supplementary Video S1). An additional advantage is that it allows us to investigate whether a change in the dynamic behaviour affects the duration of T-cell-DC interactions. In particular, if dendrites that contact a specific $\mathrm{T}$-cell retract more slowly than dendrites not making a specific contact, this prolongs the average contact duration (Figure $4 \mathrm{a}$, red lines). The competition between large numbers of fast-moving $\mathrm{T}$ cells can easily lead to an accidental loss of contact between a thin dendrite and a T-cell that is contacted at the tip of the dendrite. Our simulation results suggest that such a loss of contact is less likely to occur when the dendrite retracts with low velocity. Hence, a possible strategy of DCs that promotes long-lasting interactions between $\mathrm{T}$ cells and DCs may be that their dendrites retract slowly when they sense a specific contact (although this is not required to explain long contacts). 
a

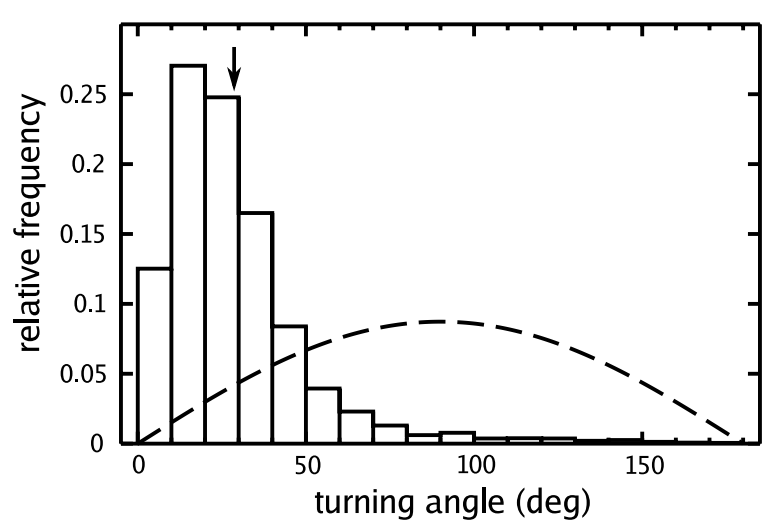

b

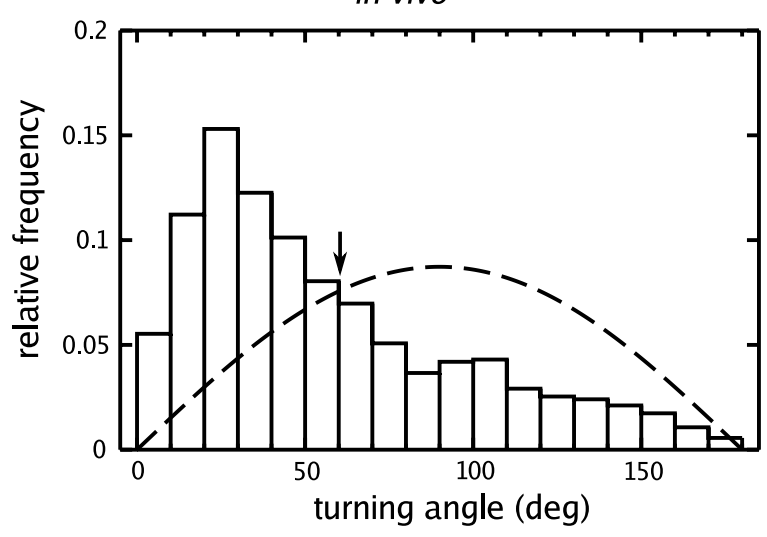

C

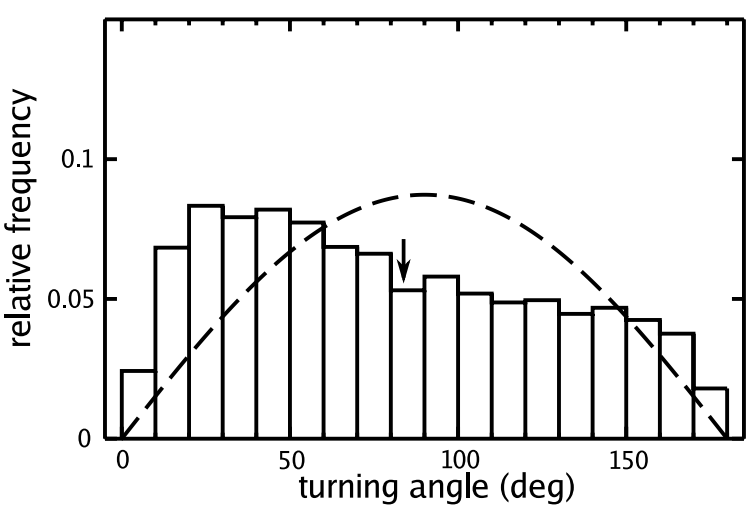

Figure 2 Persistence of T cells in silico and in vivo. (a) Distribution of T-cell turning angles in silico in the absence of antigen. ( $\mathbf{b}$ and $\mathbf{c}$ ) Distribution of T-cell turning angles in vivo in the absence (b) and presence (c) of LPSmatured DCs (data are replotted from Mempel et al. ${ }^{5}$ ). Dashed lines shows the distribution expected in the case of random turning. Arrows denote mean turning angles: (a) $28.7^{\circ}$, (b) $60.4^{\circ}$, and (c) $83.8^{\circ}$. In silico turning angles are measured each $20 \mathrm{~s}$, in vivo turning angles each $15 \mathrm{~s}$.

The duration of measurement

The typical duration of 2PM imaging experiments is only 30-60 min, which is due to current technical limitations of $2 \mathrm{PM}^{26}$ The majority of T-cell-DC interactions exceeds this duration in the phase of longlived contacts, which makes it difficult to make a good estimate on the actual mean interaction time. ${ }^{27}$ Our simulations provide an excellent tool to make such an estimate, because we can perform long-term in silico measurements (after prior fitting to short time scale data). Even in the presence of specific stop signals and high adhesion between specific $\mathrm{T}$ cells and antigen-bearing DCs, the percentage of remaining contacts keeps decaying slowly at time scales longer than $1 \mathrm{~h}$ (Figure $4 \mathrm{~b}$ ). Note that during a simulation there are no changes in any of the parameters (e.g., in adhesion preference). Hence, all losses of contact are accidental, and are due to stochastic membrane fluctuations.

In experimental plots of the percentage of remaining contacts over time, typically only interactions whose initiation and termination is observed, or that last the entire observation period, are included in the analysis. ${ }^{5}$ Our long simulations allow us to investigate how the length of the observation method affects the results of this method. First, parameters are tuned such that for a brief 1 -h observation period we reproduce the result that $80-90 \%$ of contacts remain intact after $60 \mathrm{~min}$. Next, long simulations of $10 \mathrm{~h}$ are performed at the same 'realistic' parameter settings. The percentage of contacts (during the entire 10 -h interval) lasting longer than $60 \mathrm{~min}$ is then recorded. This appears to be only $20-40 \%$ of contacts, which is much lower than the percentage measured in a brief simulation and in experiments (Figure 4a, compare lines of same colour). This difference is explained by the fact that in a brief observation window there is a bias against observing contacts of intermediate duration: their initiation or termination point tends to fall outside the observation window. Hence, although studying the percentage of remaining contacts over time is an excellent indicator for the start of the long-contact phase, it is important to realize that it reflects an inaccurate distribution of contact times.

The distributions of contact and intercontact times as measured in a 2.5-day simulation (for a parameter setting where approximately $80 \%$ of contacts remains intact in a 1-h simulation) are shown in Figure 4c. From this, the average time of specific interactions is predicted to be approximately $1.5 \mathrm{~h}$ (note that there is a large variation). Hence, although in a measurement period of $1 \mathrm{~h}$ most contacts remain intact in 2PM experiments, according to our simulations this does not translate into an average contact duration of many hours.

\section{DISCUSSION}

We demonstrated that to explain the long-lasting T-cell-DC contacts in 'phase 2' of an immune response, both the presence of stop signals transmitted by antigen-bearing DCs and a high adhesion between specific $\mathrm{T}$ cells and antigen-bearing DCs are required. In the earlier 'phase 1' of experiments, T cells do not form long-lived contacts with antigen-bearing DCs, but, in comparison to the situation without antigen, they have slightly longer contacts with those DCs, and are less motile due to a lower mean velocity ${ }^{14}$ and higher turning angles. ${ }^{5,14}$ Our simulations show that the changes in phase 1 cannot be explained by an increase in adhesion between specific T cells and antigen-bearing DCs alone. Although this does lead to a small increase in contact duration, it does not affect the motility, velocity, and turning angles of $\mathrm{T}$ cells. Our results show that the presence of stop signals transmitted by antigen-bearing DCs is able to explain all phase 1 characteristics. Hence, our simulations predict that as soon as activated, antigenbearing DCs enter the LN, they start transmitting stop signals to $\mathrm{T}$ cells. Perhaps, such signals are given regardless of specificity. This could explain the finding that the presence of LPS-matured DCs (i.e., they are activated but do not carry antigen) is sufficient to give rise to phase 1 T-cell behaviour. ${ }^{5}$ During phase 1 , the upregulation and/or activation of adhesion molecules (possibly in both $\mathrm{T}$ cells and antigenbearing DCs) as a consequence of signals integrated during brief 


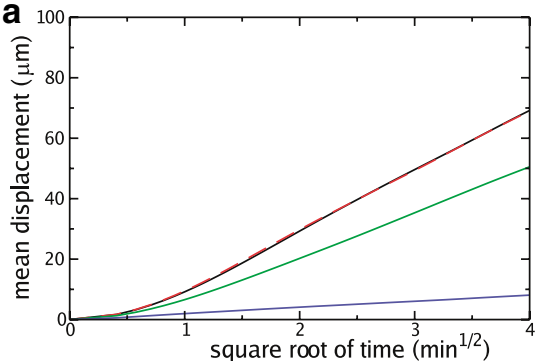

no stop signal
weak adhesion: $\gamma_{\mathrm{sT}, \mathrm{agDC}}=-2$
no stop signal
strong adhesion: $\gamma_{\mathrm{sT}, \mathrm{agDC}}=-800$

rat

stop signal

weak adhesion: $\gamma_{\mathrm{sT}, \mathrm{agDC}}=-2$

stop signal

strong adhesion: $\gamma_{\mathrm{sT}, \mathrm{agDC}}=-600$

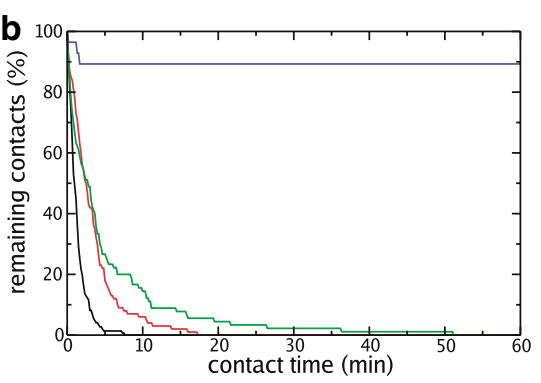

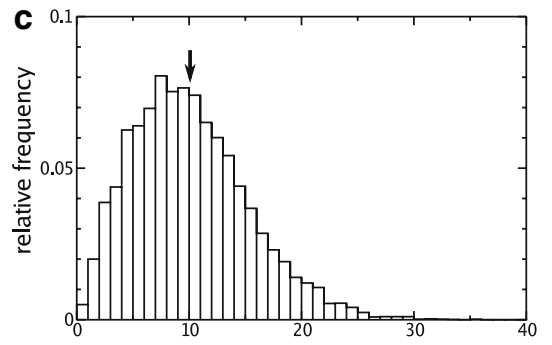
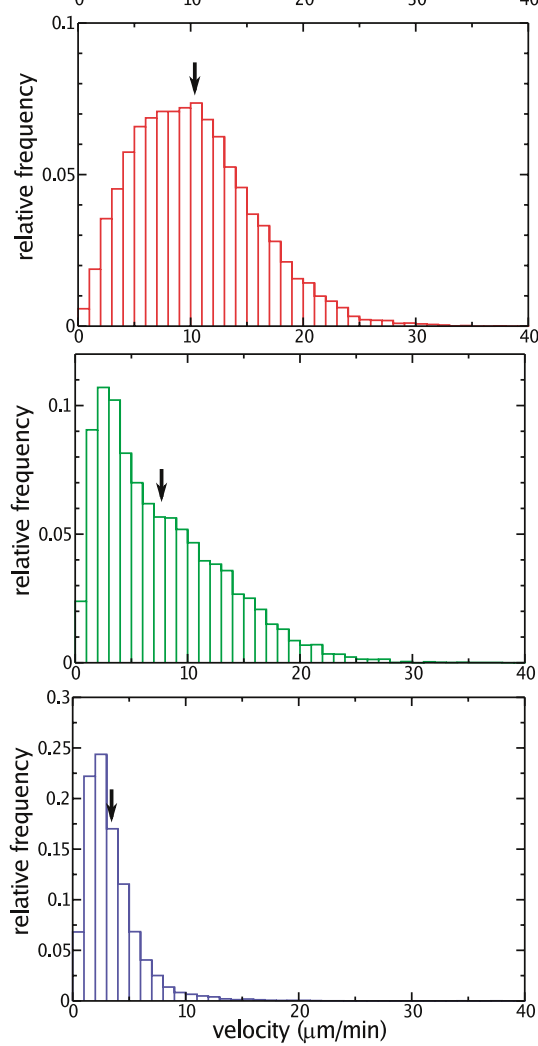
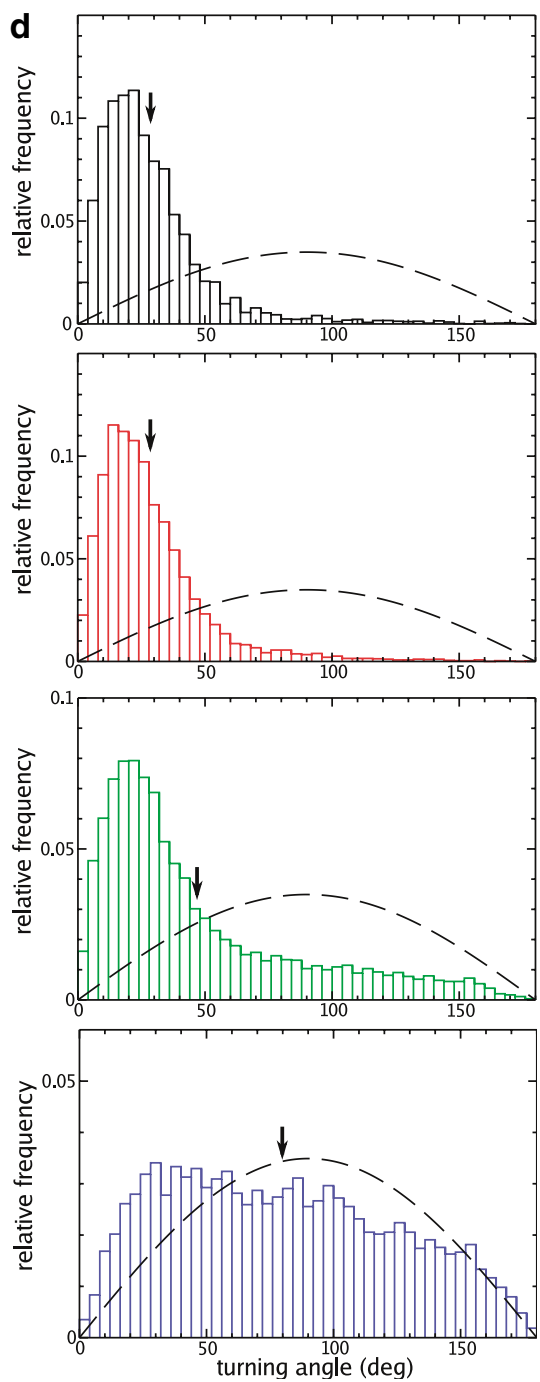

Figure 3 The effect of specific stop signals and high adhesion between specific T cells and antigen-bearing DCs on the dynamic behaviour of simulated T cells. (a) Mean displacement plots. (b) Percentage of interactions between specific T cells and antigen-bearing DCs that remain in contact after the given duration. (c) Distributions of T-cell velocities. Arrows denote mean velocities (black, $10.1 \mu \mathrm{m} \mathrm{min}^{-1}$; red, $10.4 \mu \mathrm{m} \mathrm{min}^{-1}$; green, $7.7 \mu \mathrm{m} \mathrm{min}{ }^{-1}$; and blue, $3.4 \mu \mathrm{m} \mathrm{min}^{-1}$ ). (d) Distributions of T-cell turning angles. Dashed lines show the distribution expected in case of random turning. Arrows denote mean turning angle (black, $28.7^{\circ}$; red, $28.5^{\circ}$; green, $46.9^{\circ}$; and blue, $79.8^{\circ}$ ).

interactions could enable the transition to phase 2. Furthermore, adhesion molecules concentrate in the centre of the contact zone during the formation of the IS. ${ }^{16}$ Hence, the IS may be just as important in the stabilization of contacts as in the signalling processes that lead to T-cell activation.

An abrupt increase in intracellular calcium concentration and associated loss of motility has been found in several cases: in B cells upon contact with antigen-bearing DCs in the LN T-cell area, ${ }^{28}$ in thymocytes upon interaction with stromal cells during positive selection in in vitro thymic slice preparations, ${ }^{29}$ and in T cells interacting with B cells in vitro. ${ }^{30}$ Therefore, such calcium influx may be indicative of a stop signal. ${ }^{31}$ Similar evidence for a stop signal has not yet been demonstrated for the case of specific T cells interacting with antigenbearing DCs, but seems likely. On the basis of our simulations we suggest that DCs transmit stop signals at least in phase 1 and phase 2 . In phase 3 of $\mathrm{T}$-cell priming, $\mathrm{T}$ cells have resumed their rapid migration and brief contacts with DCs. This may mean that DCs have ceased their transmission of stop signals, or that activated $\mathrm{T}$ cells no longer respond to them. It was recently shown that CTLA $-4^{+} \mathrm{T}$ cells fail to form long-lasting interactions with DCs. ${ }^{32}$ Similarly, this may be because such $\mathrm{T}$ cells are ignoring DC stop signals.

Another outcome of our simulations is that DCs are able to maintain longer contacts if they retract dendrites that sense a specific contact more slowly than other dendrites. In the densely packed LN, environment (both in reality and in our simulations) contacts may break accidentally because of random membrane fluctuations and collisions with the multitude of competing $\mathrm{T}$ cells that move around at high velocities. A DC strategy where dendritic processes retract more slowly upon recognition of a cognate interaction would facilitate the establishment of stable contacts. Although this strategy is not required to explain long-lived interactions, it is an interesting possibility that could be tested in vivo. It could also explain why most stable, specific contacts between $\mathrm{T}$ cells and DCs occur on dendrites (as reported by Miller et al. ${ }^{14}$ for $\mathrm{CD}^{+}{ }^{+} \mathrm{T}$ cells). 


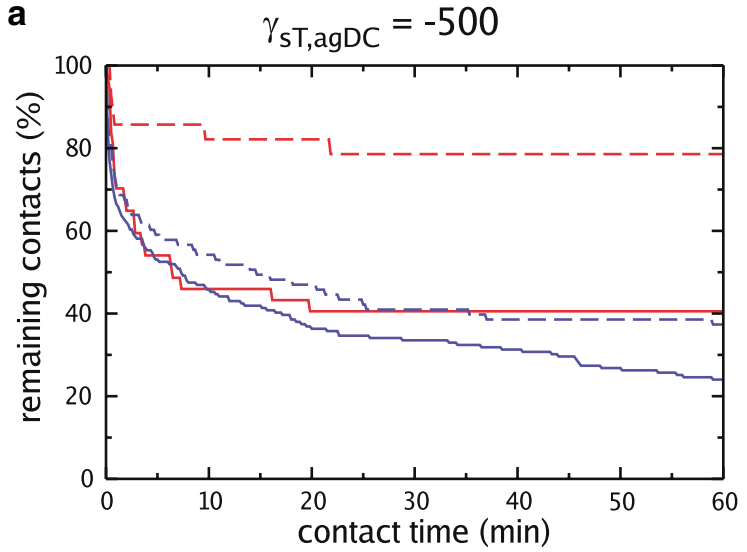

b

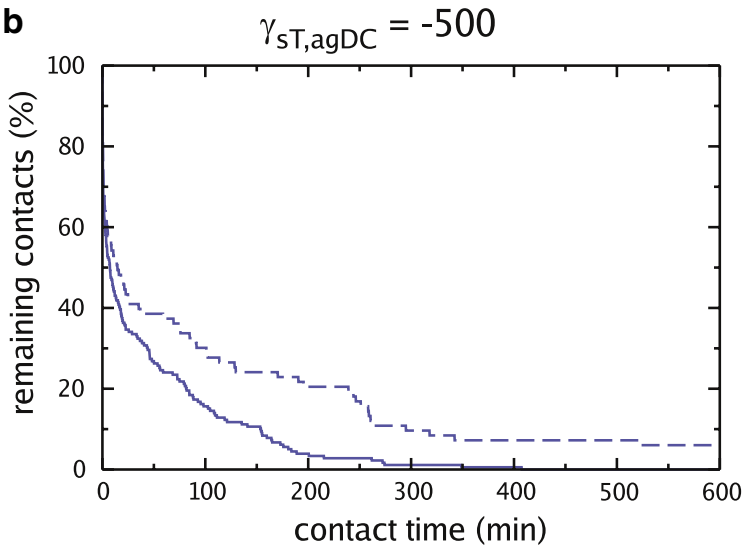

C

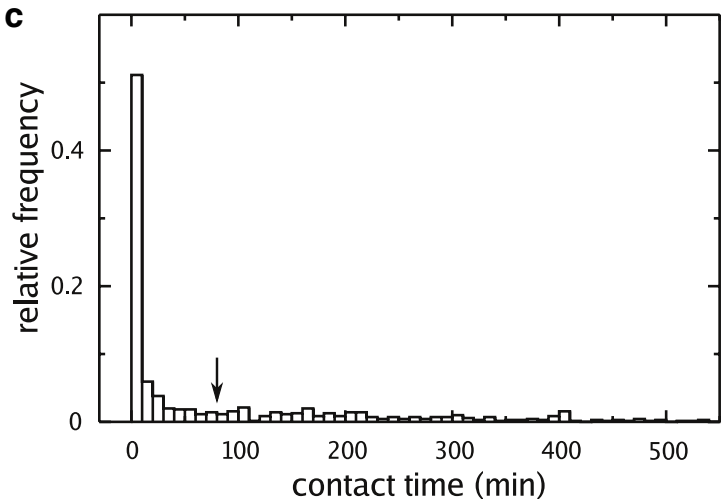

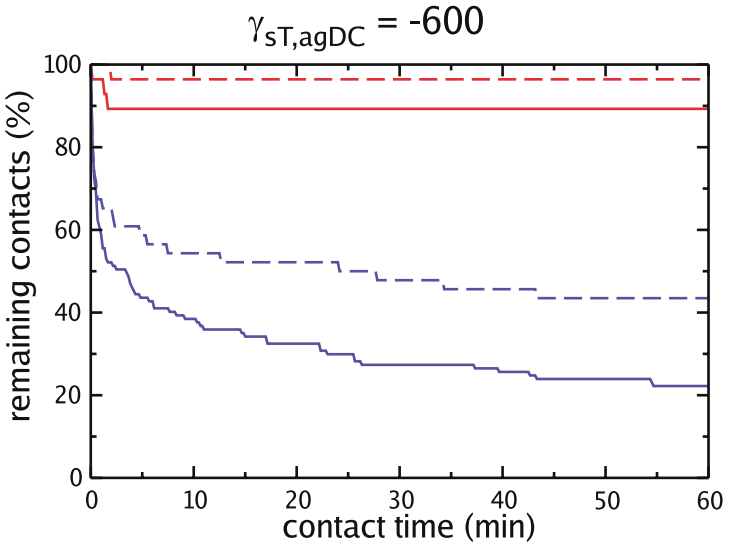
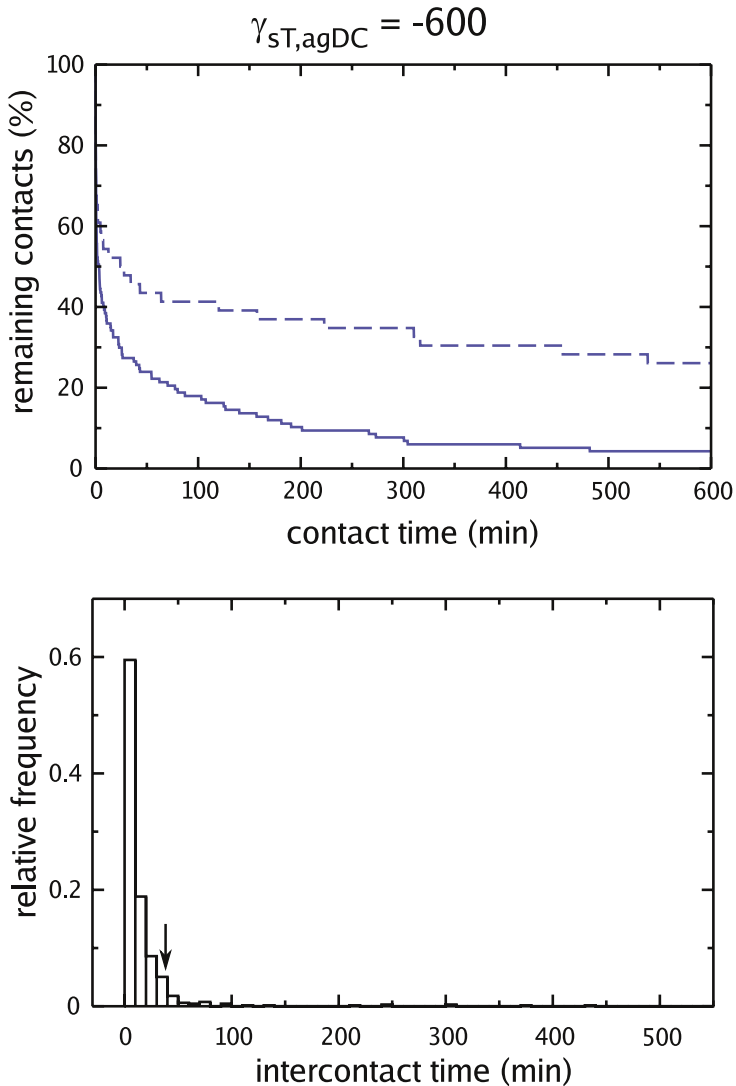

Figure 4 The effect of speed of dendrite retraction and length of measurement window on contact duration. (a and b) Percentage of contacts between specific T cells and antigen-bearing DCs that remain in contact after the given duration. Red lines show results for 1-h simulations and blue lines for 10-h simulations. Dashed lines show results for slow retraction of dendrites that sense a specific contact $\left(p_{\text {retr }}=0.1, p_{\text {retr, sp }}=0.01\right)$ and solid lines show results for the case that all dendrites retract with the same average velocity $\left(p_{\text {ret }}=p_{\text {retr,sp }}=0.1\right)$. (c) Distribution of contact times (left panel) and intercontact times (right panel) in a 2.5-day simulation. Average contact time $(80.0 \mathrm{~min})$ and intercontact time $(38.3 \mathrm{~min})$ are denoted by arrows. Notice the large variation in contact time. In all panels, only contacts whose initiation and termination is observed or that last the entire observation period are included.

In our model, we have considered that DCs maintain themselves in a more or less fixed mean position among a network of other DCs, as was shown in $2 \mathrm{PM}$ experiments. ${ }^{24}$ In addition, antigen-bearing DCs were evenly distributed over space at the start of our simulations, which prevented specific $\mathrm{T}$ cells bouncing henceforth between nearby antigen-bearing DCs. In simulations with random positioning of antigen-bearing DCs, we frequently observed many short-lived contacts because of such bouncing (not shown). Furthermore, when we did not fix the approximate mean position of DCs, this resulted in the formation of clusters involving multiple antigen-bearing DCs and specific T cells (a result of the high adhesion between these cell types). Transfer of T cells between different DCs is actually regularly observed in real LNs during the second phase of long-lived interactions. ${ }^{14}$ However, it would probably be difficult to observe fast bouncing in 2PM experiments due to the resolution of images in space and time. The formation of clusters involving multiple DCs and T cells may also occur in vivo, although the anchoring of $\mathrm{DCs}$ to the $\mathrm{RN}^{12,33,34}$ may counteract it. An additional factor that may hinder cluster formation is the polarization of T-cell receptors and adhesion molecules towards antigen-presenting cells during the formation of the IS. This could 
make $\mathrm{T}$ cells less sensitive to the establishment of other contacts at their rear end. ${ }^{30}$ For simplicity, we restrained the development of clusters of multiple DCs and T cells by keeping antigen-bearing DCs approximately evenly distributed in space.

Several models have been developed to investigate the migration of $\mathrm{T}$ cells in the early stages of an immune response. Preston et al. ${ }^{25}$ modelled this as a transport-limited chemical reaction, aiming to estimate the time for a specific T-cell to encounter an antigen-bearing DC, and the density of such DCs required for a successful immune response. This is a good example of how modelling of T-cell motility can help in drawing conclusions about the requirements for the development of immunity. Beauchemin et al. ${ }^{35}$ consider T-cell motion as a sequence of 'free runs' in random directions alternated by periods of pausing. They use this minimalist description of an unhindered random walk to find the best fit for three parameters (time and velocity of a free run, and pause time) to experimental mean displacement plots.

The above approaches do not explicitly take into account the space taken up by cells, or T-cell-DC interactions. One way to model this is to consider cells that take up a single position on a 3D lattice, an approach taken by Chakraborty et al. (unpublished results). They investigate how the speed of T-cell clonal expansion depends on various model parameters such as fraction of antigen-bearing DCs and T-cell precursor frequency. Another description of lymphocyte behaviour, on the level of individually moving and deforming cells, is provided by Meyer-Hermann et al. ${ }^{36}$ They take the shape of lymphocytes into account by describing the movement of individual subunits. Although their model is able to reproduce the default T- and B-cell behaviours such as random walk, and large velocity and shape fluctuations, the formalism does not provide a proper description of cellular interactions. As a result, realistic simulations of an entire tissue are not feasible. As we have shown previously, ${ }^{20}$ the well-established CPM model formalism ${ }^{21,22}$ provides such a realistic description of LN tissue while also taking into account cell shape and cell contacts. As a consequence, our simulations generate predictions on the level of individual cells and their interactions that can be tested in new 2PM experiments.

Using a novel method of simulating the dynamic behaviour of DC dendrites, our previous results on T-cell motility in the absence of cognate antigen ${ }^{20}$ - random walk, large velocity fluctuations, shortlived, non-specific contacts with DCs - remained similar. In addition, the distribution of in silico T-cell turning angles (Figure 2a) quantifies their directional persistence. The persistence we measure in our simulations is stronger than that measured experimentally, that is, real $\mathrm{T}$ cells take larger turns than our in silico $\mathrm{T}$ cells, yet they manage to achieve similar motility coefficients. This may be related to their strong preference to walk along the FRC network, which was discovered recently by Bajénoff et al. ${ }^{12}$ The shape of the network could force $\mathrm{T}$ cells to make large turns, but still realize large displacements. Whether the incorporation of a more realistic $\mathrm{RN}$ leads to a better match between turning angle distribution and motility coefficient awaits further investigation.

Here, we provide for the first time a model of brief and long interactions between $\mathrm{T}$ cells and DCs. Experimental tests of our model-based predictions include investigating the presence of stop signals and the expression of adhesion molecules at different time points after T-cell transfer. Further, it would be interesting to test whether the dynamic behaviour of DC dendrites changes upon sensing a specific contact, a hypothetical strategy that we pinpointed as promoting stable contacts. The ability of generating testable predictions demonstrates the suitability of our modelling approach to assist $2 \mathrm{PM}$ imaging experiments in improving our understanding of the development of immunity.

\section{METHODS}

\section{CPM}

The $\mathrm{CPM}^{21,22}$ considers a lattice where multiple connected sites (with 3D coordinates $i, j$, and $k$ ) together comprise cells of type $\tau(\sigma)$, where $\sigma$ represents the cell identification number. This has the advantage that cell have a shape, which can change over time. Sites that contact other cells, RN or ECM, have a surface energy with their direct surroundings. Cells are assumed to minimize their surface energies, and this gives rise to changes in cell configurations and movements over the course of time. To determine how an extension of a lattice site into a random neighbour, which is constantly attempted during a simulation, would change the surface energy, one calculates the so-called Hamiltonian:

$$
H=\sum_{i j k} \sum_{i^{\prime} j^{\prime} k^{\prime}} J_{\tau\left(\sigma_{i j k}\right), \tau\left(\sigma_{i^{\prime} j^{\prime}, k^{\prime}}\right)}\left(1-\delta_{\left.\sigma_{i j k}, \sigma_{i^{\prime} j^{\prime} k^{\prime}}\right)}\right)+\sum_{\sigma} \lambda_{v}\left(v_{\sigma}-V_{\sigma}\right)^{2}
$$

where the first term represents the sum of all surface energies $J$, and the second term is required to keep cells of actual volume $v$ close to their target volume $V$. Further, $\delta$ is the Kronecker delta and $\sigma_{i^{\prime} j^{\prime} k^{\prime}}$ sums over all 26 neighbours in the $3 \times 3 \times 3$ neighbourhood. The probability that a lattice site is copied to a neighbouring site is 1 if $\Delta H<0$, and $\mathrm{e}^{-(\Delta H) / T}$ otherwise, where $\Delta H$ is the change in $H$ due to the considered modification, and $T$ represents the membrane fluctuation amplitude of cells. The model as well as the measurements on cellular behaviour were implemented in the $\mathrm{C}$ programming language.

\section{Describing DC dendrites}

Experimental work has indicated that the actin-bundling fascin proteins are associated with the formation of dendritic processes, ${ }^{37}$ and are involved in the antigen presentation activity of DCs. ${ }^{38}$ Indeed, fascins generally enable growth of needle-like structures. ${ }^{39}$ We describe dendrites of in silico DCs by explicitly defining multiple ( $\left.N_{\text {bundles }}\right)$ thin actin bundles that start growing at a position 'inside' the cell, and in a random, straight direction. Each time step actin bundles extend themselves a single position into their direction of movement, provided that the site they are growing into belongs to the DC. When this is not the case, the dendrite will try again the following time step. When extension of an actin bundle fails for 20 time steps in a row, or the bundle bumps into the static RN, it will start retracting. Otherwise, the bundle retracts after a maximum growth period of 150 time steps (this determines the maximum length a dendrite can obtain, although it will generally be much shorter). Actin bundles pull themselves back in reverse order as they have grown. Retraction takes place with a single position per time step, with probability $p_{\text {retr }}$ (this parameter determines the speed of retraction). In some simulations, actin bundles associated with dendrites contacting a specific T-cell have a reduced retraction probability of $p_{\text {retr,sp. }}$. As soon as an actin bundle has pulled itself back completely, a new bundle starts to extend.

The pushing of actin bundles against the DC cell membrane is modelled by increasing the likelihood that membrane elements grow into positions adjacent to a bundle. This is performed by incorporating an extra term in $\Delta H$ : when an extension of membrane into a bundle-neighbouring site is considered, $\Delta H$ is decreased with $E_{\text {extend }}$ (this parameter determines how much DCs are inclined to extend dendrites). Furthermore, to prevent the breaking of dendrites, membrane elements adjacent to actin bundles are required to remain intact.

The initial position of antigen-bearing DCs is approximately evenly distributed in space. In most of the simulations presented in this paper, we consider DCs that are more or less fixed in a network of other DCs. ${ }^{24}$ This was achieved by restricting the starting position of new dendrites to a cubic area of five positions around the initial mean position of the cell. In simulations without fixation of DCs, new dendrites always start out from the current mean DC position, thus allowing for displacement from the initial position. Note that apart from the active extension and retraction of dendrites, DC movements are a result of membrane fluctuations alone. 


\section{Modelling T-cell motility}

$\mathrm{T}$ cells are considered to exhibit a polarity, which makes extensions of lattice sites that are approximately in the direction of the polarity more likely than other extensions. This was implemented by the following extra term in $\Delta H$ for T cells:

$$
\Delta H=-\mu \cos (\alpha)
$$

where $\mu$ is the 'directional propensity' of cells, and $\alpha$ is the angle between polarity and the considered displacement vector (the vector given by the coordinate of the position whose modification is considered and the mean position of the cell). The polarity of $\mathrm{T}$ cells is initially random, but is updated each $\Delta t$ seconds to become the displacement vector of the previous period. To describe cell turning in a realistic way, that is, abrupt directional changes are not possible, the actual $\mu$ value of a T-cell is also adjusted according to its recent displacement: $\mu=\mu_{\max } \mathrm{e}^{-\rho(1-\cos \beta)}$, where $\mu_{\max }$ is the maximum directional propensity $\mathrm{T}$ cells can obtain, $\beta$ is the angle between the displacement and target vector of the previous period, and $\rho$ determines how rapidly the directional propensity declines when a turn is occurring. The directional propensity of $\mathrm{T}$ cells is utilized to tune the average velocity of $\mathrm{T}$ cells to values similar to their experimental velocity in the absence of cognate antigen. A specific T-cell that receives a stop signal because it is in contact with an antigenbearing DC turns its directional propensity to 0 , that is, looses its directional motility. When the contact subsequently breaks, the directional propensity is 'turned on', at a value according to its recent, usually small, displacement (see the above formula).

\section{Default model parameters}

When not otherwise noted, the default parameters as described in this subsection are used. We model a wrapped cubic space (3D torus) with a length of $100 \mu \mathrm{m}$ in all three dimensions. One site of the lattice equals $1 \mu \mathrm{m}^{3}$. The space is filled with 3000 rods with a radius of $1 \mu \mathrm{m}$ and a height of $20 \mu \mathrm{m}$, which represents the RN (in total this takes up approximately $17 \%$ of the space). The following numbers of cells are considered: 30 specific T cells, 3500 non-specific $\mathrm{T}$ cells, eight antigen-bearing DCs, and 142 DCs presenting irrelevant antigen. Cells are initialized at a random position (except for antigen-bearing DCs that are evenly distributed in space), as a $27 \mu \mathrm{m}^{3}$ block, and subsequently grow to their target volumes $\left(150 \mu \mathrm{m}^{3}\right.$ for $\mathrm{T}$ cells and $1400 \mu \mathrm{m}^{3}$ for DCs). The surface area of DCs is measured as the number of direct, non-diagonal neighbours not belonging to the cell, summed for all DC positions. For the default parameter settings, the surface area attained by in silico DCs is approximately $1800 \mu \mathrm{m}^{2}$, which is close to the experimentally measured values of $1800-2400 \mu \mathrm{m}^{2}{ }^{4}$ At the mentioned settings for cell and RN density there is approximately $5 \%$ of the space left for ECM, that is, we have created a densely packed 3D space.

Surface tension between cell types $x$ and $y, \gamma_{x, p}$ is calculated from the surface energies as follows $\left.{ }^{22}: \gamma_{x, y}=J_{x, y}-\left(J_{x, x}+J_{y, y}\right) / 2\right)$. A negative surface tension means that cells of type $x$ and $y$ want to intermingle, that is, have an adhesive preference for the other cell type. Recent 2PM experiments have shown that $\mathrm{T}$ cells preferentially walk along the RN. ${ }^{12}$ Therefore, we consider all $\mathrm{T}$ cells to have a strong preference to adhere to the RN. Non-specific T cells have a small preference to adhere to DCs, which is also true for specific T cells and DCs not presenting relevant antigen. We investigate the effect of adhesion preference between specific T cells and antigen-bearing DCs $\left(\gamma_{s T, a g D C}\right)$ on cell motility and interactions, which is achieved by modifying $J_{s T, a g D C}$. Other than T-cell-DC and T-cell-RN interactions, there is no differential adhesion between in silico DCs, $\mathrm{T}$ cells, RN, and ECM. Table 1 shows the default surface energy parameters and resulting adhesion strengths (here, DC stands for both types of DCs, and T stands for both types of $\mathrm{T}$ cells). Other default parameters used are: $N_{\text {bundles }}=40, E_{\text {extend }}=20000, p_{\text {retr }}=p_{\text {retr }, s p}=0.1, \quad \mu_{\max }=6000, \Delta t=20 \mathrm{~s}, \rho=3$, $T=500$, and $\lambda=500$. Results shown are representative of several simulations.

\section{Simulation measurements}

After one Monte Carlo time step in the simulations, all positions in the lattice have been considered for updating. This corresponds to $1 \mathrm{~s}$ in real time. To allow the onset of long-lasting contacts, measurements start after an initial $5000 \mathrm{~s}$, which is defined as time 00:00 (min:s). The mean positions of $\mathrm{T}$ cells are
Table 1 Default surface energies and surface tensions

\begin{tabular}{lcccc}
\hline & T cell & $D C$ & $R N$ & $E C M$ \\
\hline T cell & $J_{T, T}=1000$ & $\gamma_{T, D C}=-2$ & $\gamma_{T, R N}=-400$ & $\gamma_{T, E C M}=0$ \\
DC & $J_{D C, T}=998$ & $J_{D C, D C}=1000$ & $\gamma_{D C, R N}=0$ & $\gamma_{D C, E C M}=0$ \\
RN & $J_{R N, T}=100$ & $J_{R N, D C}=500$ & $J_{R N, R N}=0$ & $\gamma_{R N, E C M}=0$ \\
ECM & $J_{E C M, T}=100$ & $J_{E C M, D C}=500$ & $J_{E C M, R N}=0$ & $J_{E C M, E C M}=0$ \\
\hline
\end{tabular}

Abbreviations: DC, dendritic cell; ECM, extracellular matrix; RN, reticular network.

registered every $10 \mathrm{~s}$, and are used to calculate displacements and velocities. Motility coefficients are estimated according to the formula $M=|\vec{X}|^{2} / 6 t$ (explained in Sumen et al. ${ }^{40}$ ). Interactions between $\mathrm{T}$ cells and DCs are counted as contacts when they have at least one direct (non-diagonal) neighbour. Contacts, as well as the turning angles of T cells, are registered every $20 \mathrm{~s}$.

\section{CONFLICT OF INTEREST}

The authors have no conflicting financial interest.

\section{ACKNOWLEDGEMENTS}

We thank Mark Miller for discussions on the behaviour of DC dendrites. This work was supported by HFSP grant RGP0010/2004 (JBB) and by NWO grants 863.03.007 (AFMM) and 016.048.603 (RJDB).

1 Miller MJ, Wei SH, Parker I, Cahalan MD. Two-photon imaging of lymphocyte motility and antigen response in intact lymph node. Science 2002; 296: 1869-1873.

2 Bousso P, Bhakta NR, Lewis RS, Robey E. Dynamics of thymocyte-stromal cell interactions visualized by two-photon microscopy. Science 2002; 296: 1876-1880.

3 Miller MJ, Wei SH, Cahalan MD, Parker I. Autonomous T-cell trafficking examined in vivo with intravital two-photon microscopy. Proc Natl Acad Sci USA 2003; 100: 2604-2609.

4 Miller MJ, Wei SH, Cahalan MD, Parker I. T-cell repertoire scanning is promoted by dynamics dendritic cell behavior and random T-cell motility in the lymph node. Proc Natl Acad Sci USA 2004; 101: 998-1003.

5 Mempel TR, Henrickson SE, von Andrian UH. T-cell priming by dendritic cells in lymph nodes occurs in three distict phases. Nature 2004; 427: 154-159.

6 Hugues S, Fetler L, Bonifaz L, Helft J, Amblard F, Amigorena S. Distinct T-cell dynamics in lymph nodes during the induction of tolerance and immunity. Nat Immuno 2004; 5: 1235-1242.

7 Shakhar G, Lindquist RL, Skokos D, Dudziak D, Huang JH, Nussenzweig MC et al. Stable T-cell-dendritic cell interactions precede the development of both tolerance and immunity in vivo. Nat Immunol 2005; 6: 707-714.

8 Witt CM, Raychaudhuri S, Schaefer B, Chakraborty AK, Robey EA. Directed migration of positively selected thymocytes visualized in real time. PLOS Biol 2005; 3: 1062-1069.

9 Celli S, Garcia Z, Bousso P. CD4 T-cells integrate signals delivered during successive DC encounters in vivo. J Exp Med 2005; 202: 1271-1278.

10 Zinselmeyer BH, Dempster J, Gurney AM, Wokosin D, Miller M, Ho H et al. In situ characterization of $\mathrm{CD}^{+}{ }^{+}$T-cell behavior in mucosal and systemic lymphoid tissues during the induction of oral priming and tolerance. J Exp Med 2005; 201: 1815-1823.

11 Bajénoff M, Breart B, Huang AYC, Qi H, Cazareth J, Braud VM et al. Natural killer cell behavior in lymph nodes revealed by static and real-time imaging. J Exp Med 2006; 203: 619-631.

12 Bajénoff M, Egen JG, Koo LY, Laugier JP, Brau F, Glaichenhaus N et al. Stromal cell networks regulate lymphocyte entry, migration, and territoriality in lymph nodes. Immunity 2006; 25: 989-1001.

13 Castellino F, Huang AY, Altan-Bonnet G, Stoll S, Scheinecker C, Germain RN Chemokines enhance immunity by guiding naive $\mathrm{CD}^{+} \mathrm{T}$-cells to sites of $\mathrm{CD}^{+}$ T-cell-dendritic cell interaction. Nature 2006; 440: 890-895.

14 Miller MJ, Safrina O, Parker I, Cahalan MD. Imaging the single cell dynamics of CD4 ${ }^{+}$ T-cell activation by dendritic cells in lymph nodes. J Exp Med 2004; 200: 847-856.

15 Monks CR, Freiberg BA, Kupfer H, Sciaky N, Kupfer A. Three-dimensional segregation of supramolecular activation clusters in T cells. Nature 1998; 395: 82-86.

16 Grakoui A, Bromley SK, Sumen C, Davis MM, Shaw AS, Allen PM et al. The immunological synapse: a molecular machine controlling T-cell activation. Science 1999; 285: 221-227

17 Stoll S, Delon J, Brotz TN, Germain RN. Dynamic imaging of T-cell-dendritic cell interactions in lymph nodes. Science 2002; 296: 1873-1876. 
18 Chakraborty AK, Dustin ML, Shaw AS. In silico models for cellular and molecular immunology: successes, promises and challenges. Nat Immunol 2003; 4: 933-936.

19 Witt C, Raychaudhuri S, Chakraborty A. Movies, measurement, and modeling: the three Ms of mechanistic immunology. J Exp Med 2005; 201: 501-504.

20 Beltman JB, Marée AFM, Lynch JN, Miller MJ, De Boer RJ. Lymph node topology dictates T-cell migration behaviour. J Exp Med 2007; 204 (www.jem.org/cgi/doi/ 10.1084/jem.20061278).

21 Graner F, Glazier JA. Simulation of biological cell sorting using a two-dimensional extended Potts model. Phys Rev Lett 1992; 69: 2013-2016.

22 Glazier JA, Graner F. Simulation of the differential adhesion driven rearrangement of biological cells. Phys Rev E 1993; 47: 2128-2154.

23 Katakai T, Hara T, Sugai M, Gonda H, Shimizu A. Lymph node fibroblastic reticular cells construct the stromal reticulum via contact with lymphocytes. J Exp Med 2004; 200 783-795.

24 Lindquist RL, Shakhar G, Dudziak D, Wardemann H, Eisenreich T, Dustin ML et al. Visualizing dendritic cell networks in vivo. Nat Immunol 2004; 5: 1243-1250.

25 Preston SP, Waters SL, Jensen OE, Heaton PR, Pritchard DI. T-cell motility in the early stages of the immune response modeled as a random walk amongst targets. Phys Rev $E$ 2006; 74: 011910.

26 Germain RN, Miller MJ, Dustin ML, Nussenzweig MC. Dynamic imaging of the immune system: progress, pitfalls and promise. Nat Rev Immunol 2006; 6: 497-507.

27 Breart B, Bousso P. Cellular orchestration of T-cell priming in lymph nodes. Curr Opin Immunol 2006; 18: 483-490.

28 Qi H, Egen JG, Huang AY, Germain RN. Extrafollicular activation of lymph node B cells by antigen-bearing dendritic cells. Science 2006; 312: 1672-1676.

29 Bhakta NR, Oh DY, Lewis RS. Calcium oscillations regulate thymocyte motility during positive selection in the three-dimensional thymic environment. Nat Immunol 2005; 6 : $143-151$.
30 Negulescu PA, Krasieva TB, Khan A, Kerschbaum HH, Cahalan MD. Polarity of T-cell shape, motility, and sensitivity to antigen. Immunity 1996; 4: 421-430.

31 Friedman RS, Jacobelli J, Krummel MF. Mechanisms of T-cell motility and arrest: deciphering the relationship between intra- and extracellular determinants. Semin Immunol 2005; 17: 387-399.

32 Schneider H, Downey J, Smith A, Zinselmeyer BH, Rush C, Brewer JM et al. Reversal of the TCR stop signal by CTLA-4. Science 2006; 313: 1972-1975.

33 Katakai T, Hara T, Lee J, Gonda H, Sugai M, Shimizu A. A novel reticular stromal structure in lymph node cortex: an immuno-platform for interactions among dendritic cells, T cells and B cells. Int Immunol 2004; 16: 1133-1142.

34 Sixt M, Kanazawa N, Selg M, Samson T, Roos G, Reinhardt DP et al. The conduit system transports soluble antigens from the afferent lymph to resident dendritic cells in the T-cell area of the lymph node. Immunity 2005; 22: 19-29.

35 Beauchemin C, Dixit NM, Perelson AS. Characterizing T cell movement within lymph nodes in absence of antigen. $J$ Immunol 2007 (in press).

36 Meyer-Hermann ME, Maini PK. Interpreting two-photon imaging data of lymphocyte motility. Phys Rev E 2005; 71: 061912.

37 Ross R, Ross XL, Schwing J, Langin T, Reske-Kunz AB. The actin-bundling protein fascin is involved in the formation of dendritic processes in maturing epidermal Langerhans cells. J Immunol 1998; 160: 3776-3782.

38 Al-Alwan MM, Rowden G, Lee TD, West KA. Fascin is involved in the antigen presentation activity of mature dendritic cells. J Immunol 2001; 166: 338-345.

39 Kureishy N, Sapountzi V, Prag S, Anilkumar N, Adams J. Fascins, and their roles in cell structure and function. Bioessays 2002; 24: 350-361.

40 Sumen C, Mempel TR, Mazo IB, von Andrian UH. Intravital microscopy: visualizing immunity in context. Immunity 2004; 21: 315-329.

Supplementary Information accompanies the paper on Immunology and Cell Biology website (http://www.nature.com/icb). 